\title{
NBTI Degradation in LTPS TFTs Under Mechanical Tensile Strain
}

\author{
Chia-Sheng Lin, Ying-Chung Chen, Ting-Chang Chang, Fu-Yen Jian, Wei-Che Hsu, Yuan-Jui Kuo, \\ Chih-Hao Dai, Te-Chih Chen, Wen-Hung Lo, Tien-Yu Hsieh, and Jou-Miao Shih
}

\begin{abstract}
This letter investigates the negative-bias temperature instability (NBTI) degradation of p-channel low-temperature polycrystalline-silicon thin-film transistors (LTPS TFTs) under mechanical tensile stress. Experimental results reveal that the interface state density $N_{\text {it }}$ and grain boundary trap density $N_{\text {trap }}$ of tensile-strained LTPS TFTs are more pronounced than those of unstrained LTPS TFTs. Extracted density of states and conduction activation energy $E_{a}$ both show increases due to the strained $\mathrm{Si}-\mathrm{Si}$ bonds, which implies that strained $\mathrm{Si}-\mathrm{Si}$ bonds are able to react with dissociated $H$ during NBTI stress. Therefore, NBTI degradation is more significant after tensile strain than in an unstrained condition.
\end{abstract}

Index Terms-LTPS, NBTI, tensile strained.

\section{INTRODUCTION}

$\mathbf{F}$ LEXIBLE flat-panel displays based on amorphous silicon thin-film transistors (a-Si TFTs) have been widely investigated [1], [2]. Compared with a-Si TFTs, low-temperature polycrystalline-silicon TFTs (LTPS TFTs) have higher electron mobility and driving current. Consequently, LTPS TFTs can integrate both the pixel array and peripheral circuits on flexible displays. The effect of tensile strain on these LTPS TFTs, when on flexible substrates, has been reported [3], [4], with results showing the potential to realize an advanced flexible display. However, reliability such as that measured by the negativebias temperature instability (NBTI) of p-channel LTPS TFTs under tensile strain has not been adequately clarified. This letter studies the NBTI degradation of p-channel LTPS TFTs under mechanical tensile strain. By analyzing conduction activation energy $E_{a}$ and density of states (DOS) and extracting interface state density $N_{\text {it }}$ and grain boundary trap density $N_{\text {trap }}$ after NBTI stress, the results indicate that distortion of $\mathrm{Si}-\mathrm{Si}$ bonds

Manuscript received March 25, 2011; revised April 9, 2011; accepted April 13, 2011. Date of publication May 31, 2011; date of current version June 29, 2011. This work was supported by the National Science Council under Contract NSC-99-2120-M-110-001 and Contract 97-2112-M-110-009MY3. The review of this letter was arranged by Editor A. Flewitt.

C.-S. Lin and Y.-C. Chen are with the Department of Electrical Engineering, National Sun Yat-Sen University, Kaohsiung 804, Taiwan.

T.-C. Chang is with the Department of Physics and the Center for Nanoscience and Nanotechnology, National Sun Yat-Sen University, Kaohsiung 804, Taiwan (e-mail: tcchang@mail.phys.nsysu.edu.tw).

F.-Y. Jian is with the Department of Physics, National Sun Yat-Sen University, Kaohsiung 804, Taiwan, and also with the Institute of Electronics, National Chiao Tung University, Hsinchu 300, Taiwan.

W.-C. Hsu, T.-C. Chen, W.-H. Lo, T.-Y. Hsieh, and J.-M. Shih are with the Department of Physics, National Sun Yat-Sen University, Kaohsiung 804, Taiwan.

Y.-J. Kuo and C.-H. Dai are with the Institute of Electro-Optical Engineering, National Sun Yat-Sen University, Kaohsiung 804, Taiwan.

Color versions of one or more of the figures in this letter are available online at http://ieeexplore.ieee.org.

Digital Object Identifier 10.1109/LED.2011.2144953
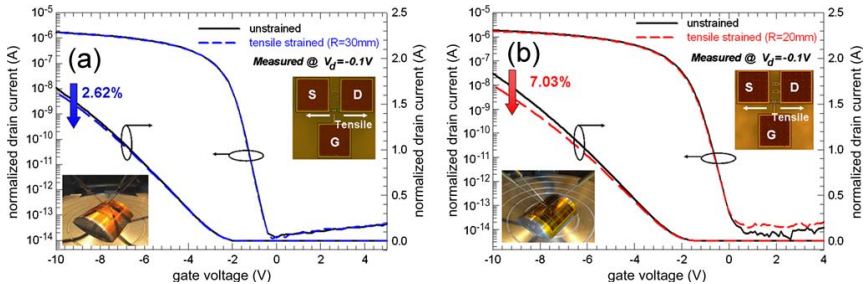

Fig. 1. (a) and (b) $I_{d}-V_{g}$ transfer characteristics with $-0.1-\mathrm{V}$ drain voltage under unstrained and mechanical-tensile-strained conditions with $R=30$ and $20 \mathrm{~mm}$.

in the poly-Si film caused by tensile strain is liable to react with the dissociated $\mathrm{H}$ under NBTI stress, resulting in more serious degradation for tensile-strained LTPS TFTs.

\section{EXPERIMENT}

In this letter, top-gate commercial p-channel LTPS TFTs without a lightly doped drain were prepared. The detailed process for producing LTPS TFTs has been reported in our previous paper [5]. The TFTs studied in this letter were 6 and $10 \mu \mathrm{m}$ in length and width, respectively. During the stress operation, the device was stressed with a gate voltage of $-20 \mathrm{~V}+$ threshold voltage for $1000 \mathrm{~s}$ for both the unstrained and mechanical-tensile-strained TFTs, whereas the source and the drain were both grounded at $125{ }^{\circ} \mathrm{C}$. Tensile strain along the channel length was introduced by mechanical bending as follows: The thickness of the glass substrate was reduced from 600 to $50 \mu \mathrm{m}$ using a Struers RotoPol-21 polisher. Subsequently, the glass substrate was adhered to a metal foil and then fixed on a bending holder, as shown in the insets of Fig. 1(a) and (b). Therefore, the tensile stress applied on the sample is uniform. The strain on the top surface can be calculated by [6]

$$
\varepsilon_{\mathrm{top}}=\left(\frac{d_{f}+d_{s}}{2 R}\right) \frac{\left(1+2 \eta+\chi \eta^{2}\right)}{(1+\eta)(1+\chi \eta)}
$$

where $\eta=d_{f} / d_{s}$, and $\chi=Y_{f} / Y_{s}$, with $d_{f}$ and $d_{s}$ being the silicon film and glass substrate thicknesses, respectively. The Young's modulus of silicon film (111) orientation and glass substrate for both $Y_{f}$ and $Y_{s}$ are $170 \mathrm{GPa} . \varepsilon_{\mathrm{top}}$ was calculated as $0.08 \%$ and $0.12 \%$ for curvature radii $R$ of 30 and $20 \mathrm{~mm}$, respectively.

\section{Results AND Discussion}

Fig. 1(a) and (b) shows the normalized $I_{d}-V_{g}$ transfer characteristics under unstrained and mechanical-tensile-strained conditions with $R=30$ and $20 \mathrm{~mm}$. Here, the normalized $I_{D}$ is defined as $I_{D} /(W / L)$. The on-current decreases when 


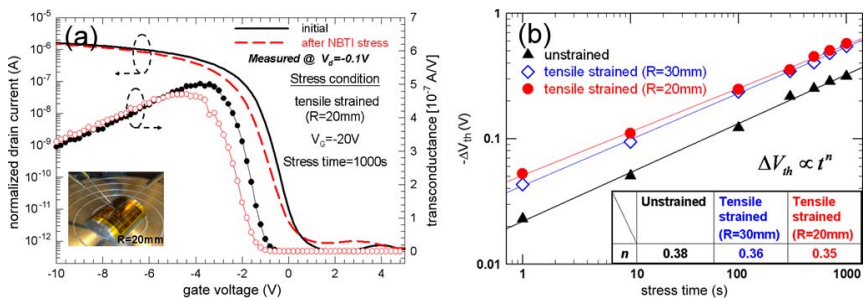

Fig. 2. (a) $I_{d}-V_{g}$ transfer characteristics with $-0.1-\mathrm{V}$ drain voltage before and after NBTI stress after mechanical tensile strain. (b) $-\Delta V_{\text {th }}$ with stress time under tensile-strain NBTI stress.

tensile strain is applied to the device, with degradation at $R=$ $30 \mathrm{~mm}$ smaller than $R=20 \mathrm{~mm}$. These results were similar to those in previous reports, indicating that the average distance between $\mathrm{Si}-\mathrm{Si}$ bonds is distorted in the poly-Si film [7], and hole mobility decreases, originating from the split between the light hole and heavy hole energy bands and an increase in mean free time on the surface orientation of poly-Si when the uniaxial tensile strain is parallel to the channel direction [4], [8].

Fig. 2(a) shows $I_{d}-V_{g}$ transfer characteristics before and after NBTI stress under mechanical tensile strain with $R=$ $20 \mathrm{~mm}$. Obviously, the subthreshold swing (S.S.) has degraded, and threshold voltage $V_{\text {th }}$ shifts to the negative direction after NBTI stress. In general, the $V_{\text {th }}$ shift of the TFT is caused by charge trapping or defect creation in the gate oxide. In this letter, the electric field across the gate dielectric is below $3 \mathrm{MV} / \mathrm{cm}$; the hole-trapping mechanism is not considered in this letter [9]. Fig. 2(b) shows $-\Delta V_{\text {th }}$ with stress time under unstrained and tensile strain NBTI stresses. $-\Delta V_{\text {th }}$ increases with an increase in stress time, which displays power-law dependence. The exponent factor $n$ can be extracted by the power-law relationship $\Delta V_{\mathrm{th}}=\mathrm{At}^{n}$ [10], where $n$ is $0.38-0.35$ for unstrained and tensile-strained conditions, respectively. This result indicates that the diffusion-controlled electrochemical reaction is the principal degradation mechanism for this NBTI stress [11]. Moreover, these results are similar to those of previous papers on LTPS TFTs [12]. This indicates that interface states and grain boundary traps were generated during NBTI stress.

$N_{\text {it }}$ can be extracted from the S.S. by ignoring the depletion capacitance in the active layer according to a previously determined equation [13]. Here, the S.S. is defined as half the gate voltage necessary to increase the drain current by two orders of magnitude (from $10^{-11}$ to $10^{-9} \mathrm{~A}$ ) in the subthreshold region.

Fig. 3(a) shows $N_{\text {it }}$ generation with stress time under unstrained and tensile-strained conditions for $R=30$ - and 20-mm NBTI stresses. $N_{\text {it }}$ generation increased with increasing stress time, becoming more seriously degraded under tensile strain than in unstrained environments. In addition, it can be observed that $N_{\text {it }}$ generation values with $R=30$ and $20 \mathrm{~mm}$ are almost the same, which implies that this degradation is sensitive to strain effect but is not a function of $R$. Grain boundary trap $N_{\text {trap }}$ can be estimated by the Levinson and Proano method [14]. Fig. 3(b) shows $N_{\text {trap }}$ generation with stress time for the unstrained condition and under tensile strain with $R=30$ - and 20-mm NBTI stresses. The $N_{\text {trap }}$ generation after tensile-strain NBTI stress is clearly more significant than that under the unstrained condition, and the most critical degradation occurs at
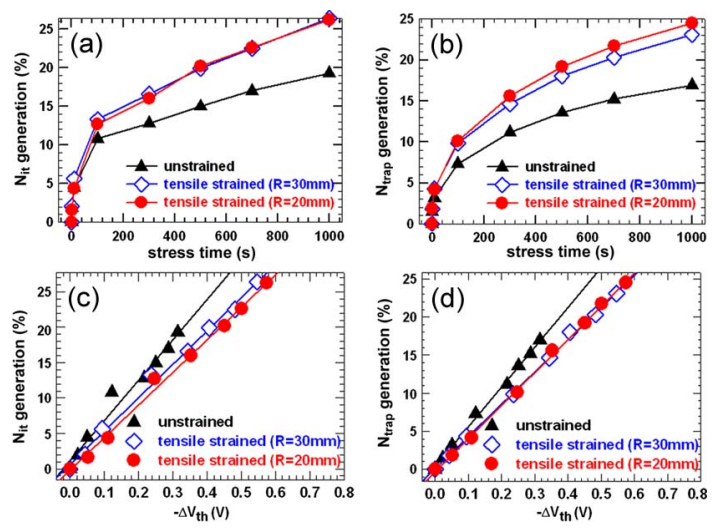

Fig. 3. (a) $N_{\text {it }}$ and (b) $N_{\text {trap }}$ generation with stress time. (c) $N_{\text {it }}$ and (d) $N_{\text {trap }}$ generation with $-\Delta V_{\text {th }}$ under unstrained and tensile-strain NBTI stresses.
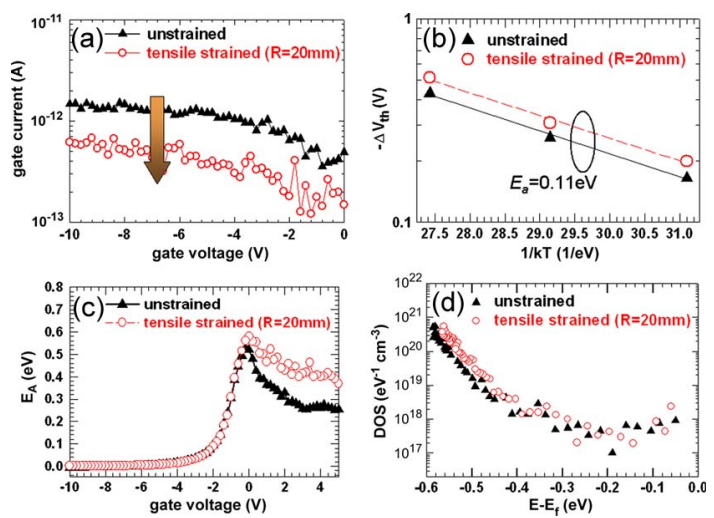

Fig. 4. (a) Gate leakage current with gate voltage. (b) $\mathrm{Si}-\mathrm{H}$ bonding energy $E_{a}$ with stress temperature for unstrained and under mechanical-tensilestrained conditions. (c) Conduction activation energy $E_{a}$ with gate voltage. (d) Effective DOS distribution for unstrained and tensile-strained TFTs.

a tensile strain of $R=20 \mathrm{~mm}$. Comparing with $N_{\text {it }}$ generation, it can be seen that the degradation in grain boundaries $N_{\text {trap }}$ is a function of $R$. In a previous report [15], the NBTI mechanism in poly-Si TFTs was explained as the inversion hole at the poly-Si/ $/ \mathrm{SiO}_{2}$ interface and grain boundaries reacting with $\mathrm{Si}-\mathrm{H}$, resulting in $\mathrm{H}$ dissociation when negative gate bias and high temperature were applied. Furthermore, the dissociated $\mathrm{H}$ species could diffuse into the gate oxide and react with the oxygen of $\mathrm{SiO}_{2}$ to form many $\mathrm{OH}$ groups bonded to $\mathrm{Si}$ atoms and Si dangling bonds. Finally, these Si dangling bonds became $N_{\text {it }}$ and $N_{\text {trap }}$ traps, and existed on the poly-Si/SiO $\mathrm{S}_{2}$ interface and grain boundaries of the channel region, respectively. On the other hand, $N_{\text {it }}$ and $N_{\text {trap }}$ degradations that originated from tensile strain with $R=20 \mathrm{~mm}$ are calculated as $4.7 \%$ and $0.6 \%$, respectively, which are smaller than the degree for strained NBTI stress. Furthermore, both $N_{\text {it }}$ and $N_{\text {trap }}$ generation were fitted with a straight line in $\Delta V_{\mathrm{th}}$, as shown in Fig. 3(c) and (d), respectively. Therefore, those serious degradations in $N_{\text {it }}$ and $N_{\text {trap }}$ are caused by not only strain effect but also NBTI stress. The previous study has reported that the hole-tunneling probability into $\mathrm{Si}-\mathrm{H}$ bonding states is probably the main origin of the enhanced NBTI degradation in strained Si p-channel metal-oxide-semiconductor field-effect transistors [16]. However, Fig. 4(a) shows that the gate leakage current decreases for the tensile-strained device; therefore, this 
mechanism can be disregarded in this letter. Another possible reason is the strain-induced vibration of the $\mathrm{Si}-\mathrm{H}$ bonding energy $E_{a}$ in the poly-Si/SiO ${ }_{2}$ interface and grain boundaries. Fig. 4(b) shows $E_{a}$ with stress time for unstrained and under tensile-strain NBTI stress and is about $0.11 \mathrm{eV}$ for both strained and unstrained devices, suggesting that larger $N_{\text {it }}$ and $N_{\text {trap }}$ generations in strained NBTI stress are not contributed by $E_{a}$ at the poly-Si/ $\mathrm{SiO}_{2}$ interface and grain boundaries. In order to realize the NBTI degradation mechanism after mechanical tensile strain, conductance activation energy $E_{a}$ and DOS were extracted. Here, $E_{a}$ is the band gap between the valence band edge and the energy of grain boundary states [14], as shown in Fig. 4(c). $E_{a}$ is observed to be greater for tensile-strained TFTs, indicating a higher band tail DOS. Fig. 4(d) shows the DOS distribution in the device channel extracted by field-effect conductance methods [17]. As can be seen, only the tail states increase slightly for the tensile-strained TFTs and coincide with $E_{a}$, which implies that the length or angle of the $\mathrm{Si}-\mathrm{Si}$ bonds has been changed, resulting in an increase in the strained $\mathrm{Si}-\mathrm{Si}$ bonds in the poly-Si channel [18]. Moreover, these strained $\mathrm{Si}-\mathrm{Si}$ bonds are able to react with $\mathrm{H}$ released from a $\mathrm{Si}-\mathrm{H}$ bond to create two defects in the reaction [19], i.e.,

$$
\mathrm{Si}-\mathrm{H}+(\mathrm{Si}-\mathrm{Si})_{s} \rightarrow \mathrm{Si}^{*}+\mathrm{Si}-\mathrm{H}+\mathrm{Si}^{*}
$$

where $(\mathrm{Si}-\mathrm{Si})_{s}$ and $\mathrm{Si}^{*}$ represent strained silicon and dangling bonds, respectively. The $\mathrm{Si}-\mathrm{H}$ represents hydrogen depassivation and is assumed to occur in the poly- $\mathrm{Si} / \mathrm{SiO}_{2}$ interface and grain boundary. Combining (3) and the NBTI degradation model for LTPS TFTs [15], strain-assisted NBTI degradation can be written as follows:

$$
\begin{aligned}
h^{+}+ & (\mathrm{Si}-\mathrm{Si}+\mathrm{Si}-\mathrm{H})_{s}(\text { grain boundary and interface }) \\
& +\mathrm{Si}-\mathrm{O}-\mathrm{Si} \equiv(\text { gate oxide }) \\
\rightarrow & \left(2 \mathrm{Si}^{*}+\mathrm{Si}-\mathrm{H}\right)_{s}(\text { grain boundary and interface }) \\
& +\mathrm{Si}^{+}(\text {fixed oxide charge })+\equiv \mathrm{Si}-\mathrm{OH}
\end{aligned}
$$

where $h^{+}$is the hole from the inversion layer. As can be seen, $\mathrm{Si}^{*}$ and $\mathrm{Si}-\mathrm{H}$ that originate from $(\mathrm{Si}-\mathrm{Si})_{s}$ are generated by dissociated $\mathrm{H}$ under NBTI stress, and these $\mathrm{Si}-\mathrm{H}$ bonds will further react with the hole or other distorted $\mathrm{Si}-\mathrm{Si}$ bonds, resulting in more $N_{\text {trap }}$ and $N_{\text {it }}$ in the poly-Si film during NBTI stress. Therefore, device degradation is more significant under tensile strain than for the unstrained condition.

\section{CONCLUSION}

This letter has studied NBTI degradation of p-channel LTPS TFTs under mechanical tensile strain. Experimental results have indicated that interface state density $N_{\text {it }}$ and grain boundary trap density $N_{\text {trap }}$ of LTPS TFTs in tensile strain are more significant after NBTI stress than for an unstrained condition. Extracted conduction activation energy $E_{a}$ and DOS have revealed that the numbers of the DOS and value of $E_{a}$ increase due to the strained $\mathrm{Si}-\mathrm{Si}$ bonds after tensile strain. Therefore, strain-induced weak $\mathrm{Si}-\mathrm{Si}$ bonds are able to react with dissociated $\mathrm{H}$ during NBTI stress, resulting in $N_{\text {trap }}$ and $N_{\text {it }}$ in poly-Si film. Consequently, NBTI degradation in tensile-strained LTPS TFTs is more pronounced than for the unstrained condition.

\section{REFERENCES}

[1] S. H. Won, J. K. Chung, C. B. Lee, H. C. Nam, J. H. Hur, and J. Jang, "Effect of mechanical and electrical stresses on the performance of an a-Si:H TFT on plastic substrate," J. Electrochem. Soc., vol. 151, no. 3, pp. G167-G170, Jan. 2004.

[2] M. C. Wang, T. C. Chang, P.-T. Liu, S. W. Tsao, and J. R. Chen, "Analysis of parasitic resistance and channel sheet conductance of a-Si:H TFT under mechanical bending," Electrochem. Solid-State Lett., vol. 10, no. 3, pp. J49-J51, Jan. 2007.

[3] C. F. Huang, Y.-J. Yang, C.-Y. Peng, F. Yuan, and C. W. Liu, "Mechanical strain effect of n-channel polycrystalline silicon thin-film transistors," Appl. Phys. Lett., vol. 89, no. 10, pp. 103 502-1-103 502-3, Sep. 2006.

[4] P. C. Kuo, A. Jamshidi-Roudbari, and M. Hatalis, "Effect of mechanical strain on mobility of polycrystalline silicon thin-film transistors fabricated on stainless steel foil," Appl. Phys. Lett., vol. 91, no. 24, pp. 243 507-1243 507-3, Dec. 2007.

[5] C. S. Lin, Y.-C. Chen, T.-C. Chang, H.-W. Li, S.-C. Chen, W.-C. Hsu, F.-Y. Jian, T.-C. Chen, and Y.-H. Tai, "Transient effect assisted NBTI degradation in p-channel LTPS TFTs under dynamic stress," J. Electrochem. Soc., vol. 158, no. 1, pp. H10-H14, 2011.

[6] Z. Suo, E. Y. Ma, H. Gleskova, and S. Wagner, "Mechanics of rollable and foldable film-on-foil electronics," Appl. Phys. Lett., vol. 74, no. 8, pp. 1177-1179, Feb. 1999.

[7] J. H. Cheon, J. H. Bae, and J. Jang, "Flexibility study of high-performance LTPS-TFT on flexible metal foil," in Proc. SID, 2007, pp. 272-275.

[8] I. H. Peng, P.-T. Liu, and T.-B. Wu, "Effect of bias stress on mechanically strained low temperature polycrystalline silicon thin film transistor on stainless steel substrate," Appl. Phys. Lett., vol. 95, no. 4, pp. $041909-$ 1-041 909-3, Jul. 2009.

[9] C. F. Weng, T.-C. Chang, H.-P. Hsieh, S.-C. Chen, W.-C. Hsu, W.-C. Kuo, and T.-F. Young, "Self-heating-induced negative bias temperature instability in poly-Si TFTs under dynamic stress," Electrochem. Solid-State Lett., vol. 11, no. 8, pp. H207-H209, May 2008.

[10] A. T. Krishnan, V. Reddy, and S. Krishnan, "Impact of charging damage on negative bias temperature instability," in IEDM Tech. Dig., 2001, p. 865 .

[11] A. E. Islam, H. Kufluoglu, D. Varghese, S. Mahapatra, and M. A. Alam, "Recent issues in negative-bias temperature instability: Initial degradation, field dependence of interface trap generation, hole trapping effects, and relaxation," IEEE Trans. Electron Devices, vol. 54, no. 9, pp. $2143-$ 2154, Sep. 2007.

[12] C. S. Lin, Y.-C. Chen, T.-C. Chang, H.-W. Li, W.-C. Hsu, S.-C. Chen, Y.-H. Tai, F.-Y. Jian, T.-C. Chen, K.-J. Tu, H.-H. Wu, and Y.-C. Chen, "Degradation of low temperature polycrystalline silicon thin film transistors under negative bias temperature instability stress with illumination effect," J. Electrochem. Soc., vol. 157, no. 2, pp. J29-J33, Jan. 2010.

[13] C. A. Dimitriadis, P. A. Coxon, L. Dozsa, L. Papadimitriou, and N. Economou, "Performance of thin-film transistors on polysilicon films grown by low-pressure chemical vapor deposition at various pressures," IEEE Trans. Electron Devices, vol. 39, no. 3, pp. 598-606, Mar. 1992.

[14] R. E. Proano, R. S. Misage, and D. G. Ast, "Development and electrical properties of undoped polycrystalline silicon thin-film transistor," IEEE Trans. Electron Devices, vol. 36, no. 9, pp. 1915-1922, Sep. 1989.

[15] C. Y. Chen, J.-W. Lee, S.-D. Wang, M.-S. Shieh, P.-H. Lee, W.-C. Chen, H.-Y. Lin, K.-L. Yeh, and T.-F. Lei, "Negative bias temperature instability in low-temperature polycrystalline silicon thin-film transistors," IEEE Trans. Electron Devices, vol. 53, no. 12, pp. 2993-3000, Dec. 2006.

[16] T. Irisawa, T. Numata, E. Toyoda, N. Hirashita, T. Tezuka, N. Sugiyama, and S.-I. Takagi, "Physical understanding of strain-induced modulation of gate oxide reliability in MOSFETs," IEEE Trans. Electron Devices, vol. 55, no. 11, pp. 3159-3166, Nov. 2008.

[17] G. Fortunato, D. B. Meakin, P. Migliorato, and P. G. Le Combers, "Fieldeffect analysis for the determination of gap-state density and Fermilevel temperature dependence on polycrystalline silicon," Philos. Mag. $B$, vol. 57 , no. 5 , pp. $573-586,1988$.

[18] N. H. Nickel, W. B. Jackson, and N. M. Johnson, "Light-induced creation of metastable paramagnetic defects in hydrogenated polycrystalline silicon," Phys. Rev. Lett., vol. 71, no. 17, pp. 2733-2736, Oct. 1993.

[19] R. A. Street and K. Winer, "Defect equilibria in undoped a-Si:H," Phys. Rev. B, vol. 40, no. 9, pp. 6236-6249, Sep. 1989. 